\title{
EFICIENCIA EN EL SECTOR FINANCIERO COLOMBIANO EN EL PERIODO 1995-2008
}

\author{
ALEJANDRO RAMÍREZ VIGOYA*" \& LAURA CRISTANCHO \\ UNIVERSIDAD MILITAR NUEVA GRANADA
}

Recibido/Received/Recebido: 17/03/2010 - Aceptado/ Accepted/Aprovado: 12/09/2010

\begin{abstract}
Resumen
En este trabajo se comprueba la eficiencia de los quince bancos comerciales de Colombia entre los años 1995 y 2008. La eficiencia se mide a través de los retornos de los factores de producción, en este caso de los préstamos y los salarios sobre los ingresos de los bancos. Como modelo teórico se utilizan funciones Cobb-Douglas y como modelo econométrico se utilizan datos panel. Se analizan los retornos en modelos de efectos fijos, ya que la muestra es toda la población de bancos comerciales en Colombia. Los modelos que se analizan se corren con efectos por año y con efectos por banco. Uno de los resultados interesantes es que el sector bancario en el periodo analizado presenta rendimientos constantes de escala. Se presenta menor eficiencia de todos los bancos en los años siguientes a la recesión de 1999.
\end{abstract}

Palabras clave: Instituciones financieras, Mercado financiero, Servicios financieros

\section{EFFICIENCY IN THE COLOMBIAN FINANCIAL SECTOR IN THE PERIOD 1995-2008}

\begin{abstract}
In this paper the efficiency of fifteen commercial Banks of Colombia during the period 1995 and 2008 is proved. The efficiency is measured through production factors returns, in this case loans and salaries on bank incomes. As theoretical model Cobb-Douglas functions are used, and data panel is used as econometric model. Returns are analyzed in fixed effects model, because the sample is the total of the population of commercial banks in Colombia. The models analyzed are made with effects per year and per bank. One of the interesting results is that banking sector during the analyzed period presents constant returns to scale. There is less efficiency in every bank after 1999 recession period.
\end{abstract}

Key words: Financial institutions, financial market, financial services.

Este artículo es un primer resultado en la línea de investigación sobre políticas públicas del grupo de investigación del Centro de Investigaciones Económicas (CIE), de la Universidad Militar Nueva Granada.

* Profesor de Planta. Universidad Militar Nueva Granada. Contacto: alejandro.ramirez@unimilitar.edu.co

"** Profesora de Cátedra. Universidad Militar Nueva Granada. Contacto: lauracristancho@gmail.com 


\title{
EFICIÊNCIA NO SETOR FINANCEIROENTRE (1995-2008)
}

\begin{abstract}
Resumo
Nestetrabalho se demonstra a eficiência dos quinze bancos comerciais na Colômbia entre 1995 e 2008. A eficiência é medida pelos retornos dos fatores de produção, neste caso de empréstimos e salários dos bancos. Como modelo teórico se utiliza funções Cobb-Douglas; como modelo econométrico, os dados em painel. Analisam-se os retornos em modelos de efeitos fixos, porque a amostra é toda a população de bancos comerciais na Colômbia. Os modelos analisados se executam com efeitos por ano e por banco. Um resultado interessante é que o setor bancário apresenta retornos de escala constantes no período em análise. Os bancos mostram baixa eficiência nos anos seguintes à recessão de 1999.
\end{abstract}

Palavras-chave: Instituições financeiras, mercado financeiro, serviços financeiros.

Ramírez, A. \& Cristancho, L. (2010) Eficiencia en el sector financiero en el periodo 1995-2008. En: Revista de la Facultad de Ciencias Económicas de la Universidad Militar Nueva Granada. rev. fac.cienc.econ, XVIII (2)

JEL: G2

\section{Introducción}

Durante los últimos años del siglo XX y los primeros del XXI, el sistema financiero colombiano sufrió grandes choques, tanto externos como internos. Los choques externos provinieron de la crisis financiera mundial y los internos de la defensa de la banda cambiaria, lo cual creó el proceso desinflacionario fortuito y el decrecimiento de la economía (Sarmiento \& Ramírez, 2005). Dada la importancia del sector bancario como generador del crédito interno, se creó el Fondo de Garantías Financieras (Fogafin) para garantizarle al público en general los recursos que poseen en los bancos, ante futuras crisis financieras. Así los establecimientos de crédito se adaptaron a las nuevas necesidades y regulaciones que se establecieron en el país, pero sufrieron durante varios años los efectos negativos de los choques externos e internos.

Debido a lo anterior, el objetivo principal de esta investigación es encontrar los niveles de Competencia y Eficiencia en el sector bancario en Colombia, para el período 1995-2008, basados en la metodología utilizada por Bailey y otros (2002). Es importante este período, dadas las reformas y cambios estructurales del sector, como respuesta de los choques, tanto externos como internos, durante los últimos años del siglo XX y los primeros del XXI.

El presente trabajo se orienta hacia la existencia de economías de escala en el sector bancario, medido a través de los retornos o rendimientos de los factores de producción sobre el producto final del sector bancario, que en este caso serán los ingresos. El trabajo se sustenta en la estimación de una función de producción Cobb-Douglas para el sector financiero, en donde se cuantifica la elasticidad de los ingresos ante cambios en los niveles de los factores.

Como factor capital se utilizan los préstamos bancarios y como factor trabajo se utiliza una variable proxy, los sueldos y honorarios, que en teoría están altamente correlacionados con el número de empleados. Como variable explicada se utiliza los ingresos e intereses bancarios. ${ }^{1} \mathrm{Al}$ analizar las elasticidades del capital y del trabajo y, por otro lado, los rendimientos o retornos de estos sobre el ingreso,

1 Los retornos de los factores se analizan a través de las elasticidades independientes de los factores. La suma de las elasticidades determinan los rendimientos a escala de los factores. 
se pueden hacer algunas inferencias estadísticas interesantes como los retornos a escala por año o los retornos por banco.

Como modelo teórico se utiliza una función de producción Cobb-Douglas ${ }^{2}$, que tiene la ventaja de que es fácilmente linealizable $\mathrm{y}$, por otro lado, se puede calcular utilizando logaritmos y con datos longitudinales $^{3}$. Como modelo econométrico se utiliza un Panel de datos ${ }^{4}$, ya que se tienen datos de 15 bancos para trece años, es decir, series de tiempo y corte transversal.

En la primera parte del trabajo se analizan algunos antecedentes de investigaciones de eficiencia en el sector financiero, tanto de eficiencia en el uso de factores de producción, como de la eficiencia en la minimización de los costos de producción. En la segunda parte se explica cuáles son los datos que se tomaron para el análisis y el por qué de los datos utilizados en las regresiones. Las siguientes secciones se refieren a los modelos tanto teóricos como econométricos utilizados y corridos.En la quinta sección se analiza los resultados de los modelos y se hace inferencia estadística a partir de las regresiones. En la última parte se llega a las conclusiones finales y a las recomendaciones para los siguientes trabajos.

\section{Antecedentes}

En la literatura existen algunos trabajos que abordan, desde diferentes perspectivas, el tema del desempeño de la economía y, especialmente, del sector financiero durante los años de crisis. Dentro de los trabajos en Colombia que se enfocan en el importante papel del sector financiero, como canalizador de recursos de unos agentes hacia sectores más productivos en donde existe baja liquidez, es importante mencionar el de Janna (2003), $y$ en la importancia del sector como generador de crecimiento económico están los de Friffill (2003), (2005), Carvajal \& Zuleta (1997) y Terada (2002).
En particular, la eficiencia en el manejo operativo de los bancos puede medirse por la capacidad de reducir los costos asociados a la oferta de un producto, como en los trabajos de Bernal \& Herrera (1983), Suescún (1987), Ferrufino (1991), Castro (2001) y Berrio \& Muñoz (2005). En la medida en que estos costos sean menores, las utilidades del banco mejorarán. Según Bernal (2007), la reducción del número de bancos en Colombia se debe al deseo de estos por mantenerse en el mercado con utilidades altas, conduciéndolos de manera estratégica a fusionarse, mejorando la eficiencia del sector. Recientemente fusiones importantes como la de Bancolombia-Conavi (2005), Davivienda-Bancafé (2007), Banco AgrarioBanco del Estado (2001), no se han tenido en cuenta en estudios recientes hechos para Colombia.

A nivel tanto nacional como internacional hay trabajos importantes que relacionan la integración financiera con la eficiencia financiera como los de Gómez (2001) y Chai \& Rhee (2005), en los cuales llegan a la conclusión de que la integración financiera en general aumentó la liquidez del sector, más que si estuvieran en mercados segregados, aumentó la competitividad, disminuyó los costos de transacción y aumentó los incentivos para la innovación.

Es importante mencionar los trabajos que relacionan la desregulación bancaria con la eficiencia del sector, uno de los cuales es el de Wu (2003), en el cual la desregulación bancaria fue importante para el desempeño de los bancos tanto individualmente como en conjunto en Australia. Un trabajo que utiliza la misma metodología aplicada en ésta investigación, es el de Bailey y otros (2002). En ése trabajo se analiza si la ineficiencia en Thailandia en el sector financiero fue la causa de la crisis de 1997 y estudian los retornos del capital y el trabajo sobre el ingreso con dummies por banco, llegando a la conclusión de que hay diferencias significativas en las eficiencias según el banco.

\footnotetext{
Es la función más utilizada para medir elasticidades y rendimientos a escala.

Los modelos en logaritmos calculan elasticidades.

Se utiliza para datos longitudinales.
} 


\section{Datos}

Los datos que se utilizan en este trabajo se tomaron de la Superintendencia Financiera ${ }^{5}$. Los bancos que se tomaron como muestra son realmente todos los bancos comerciales en Colombia: ABN Amrobank, Banco de Colombia, Banco de Bogotá, Banco de Crédito (Helms Bank), Banco Popular, Banco Agrario, Banco Bilbao Vizcaya Argentaria, Citibank, Hongkong and Shanghai Banking Corporation (HSBC), Banco Santander, Banco AV Villas, Banco Caja Social Colmena BCSC, Banco Colpatria, Banco de Occidente y Banco Sudameris.

Para todos los bancos se tomaron datos desde 1995 hasta 2008, excepto para el Banco Agrario que se tomaron datos desde 1999 y para el Banco Colpatria desde 1998. Las variables que se tomaron para los bancos son: por un lado ingresos e intereses como variable explicada $\mathrm{u}$ output, $\mathrm{y}$ por otro lado como activos se tomaron los préstamos y sueldos y honorarios como variables explicativas o inputs.

Los datos fueron deflactados con base $1998^{6}$; es decir para su utilización en los modelos econométricos los datos tienen que estar en términos reales o constantes. Aunque los datos se pueden tomar en términos nominales o corrientes. Los resultados no cambian si se toman los datos reales o nominales, ya que como las regresiones son lineales y por propiedades de estas funciones da exactamente lo mismo con variables deflactadas o sin deflactar.

Se utiliza la variable sueldos y honorarios como una proxy $^{7}$ del número de trabajadores, ya que por un lado es muy complicado encontrar el número de trabajadores por año y por banco y, por otro lado, asumimos el supuesto de que el número de trabajadores y el valor de sus sueldos están positiva y perfectamente correlacionados.

\section{Modelo Económico}

Siguiendo la metodología utilizada por Bailey y otros (2002), el modelo económico y econométrico que se utiliza en este trabajo es la muy conocida función Cobb-Douglas, que se utiliza principalmente a nivel microeconómico, pero también a nivel macroeconómico, para explicar la relación entre factores de producción y el producto final de una empresa, de una industria, de un sector económico o de la economía agregada, donde se relacionan los factores de producción agregados como capital físico y capital humano con el producto interno bruto de un país.

En este trabajo se utiliza una función Cobb-Douglas de la forma:

$$
\mathrm{Y}=\mathrm{AK} \alpha \mathrm{L} \beta
$$

Donde $Y$ es el output que se requiera explicar según el modelo, A es generalmente el nivel tecnológico, $\mathrm{K}$ es el stock de capital físico, L es el trabajo ( $\mathrm{K}$ y $\mathrm{L}$ representan los factores de producción conocidos también como los inputs). Por otro lado $\alpha$ es la elasticidad del stock de capital y $\beta$ es la elasticidad del trabajo.

La demostración de que $\alpha$ y $\beta$ son elasticidades es ampliamente conocida y no merece mayor explicación ${ }^{8}$. La función Cobb-Douglas tiene la ventaja de que se puede linealizar fácilmente aplicando logaritmos naturales a las variables, y encontrando las elasticidades. También se pueden aplicar modelos econométricos a la función linealizada. La función (1) linealizada queda de la forma general:

$$
\operatorname{LnY}=\operatorname{LnA}+\alpha \operatorname{LnK}+\beta \operatorname{LnL}^{9}
$$

Los datos se toman del PyG de cada una de las empresas.

6 El cálculo de las regresiones se pueden tomar con todos los datos en términos nominales (corrientes), o con todos los datos en términos reales (constantes). Los resultados econométricos no cambian por las propiedades de las funciones lineales.

7 Una variable proxy se supone que está altamente correlacionada con la variable que reemplaza. En este caso los sueldos se suponen correlacionados con el número de trabajadores.

8 Se aplica la fórmula de elasticidad: $\varepsilon \mathrm{K}=(\partial \mathrm{Y} / \partial \mathrm{K})^{*}(\mathrm{~K} / \mathrm{Y}) \circ \varepsilon \mathrm{L}=(\partial \mathrm{Y} / \partial \mathrm{L})^{*}(\mathrm{~L} / \mathrm{Y})$, que aplicándola a $(1)$ da como resultado $\varepsilon \mathrm{K}=\alpha, \varepsilon \mathrm{L}=\beta$

9 Al aplicar en (2) las derivadas, da como resultado que $(\partial \mathrm{LnY} / \partial \mathrm{LnK})=\alpha$ y $(\partial \mathrm{LnY} / \partial \mathrm{L} n \mathrm{~L})=\beta$ 
En la literatura teórica es importante conocer el valor de $\alpha+\beta$. Si $\alpha+\beta<1$, existen rendimientos decrecientes; esto significa que por un aumento del $1 \%$ en los factores de producción (input), el output (producción, ingresos) aumenta en una proporción menor al $1 \%$ que aumentaron los factores. $\mathrm{Si}$, por otro lado, $\alpha$ $+\beta>1$, por un aumento del $1 \%$ en los factores, el output aumenta en más de un $1 \%$. Por último si $\alpha+$ $\beta=1$, existen rendimientos constantes de escala ${ }^{10}$.

Es importante conocer el valor de $\alpha+\beta$, ya que este resultado indica si el uso de los factores (input) es eficiente o no. Esta es una forma de analizar la eficiencia en cualquier sector económico o a nivel global en una economía. Al modelo (2) se le pueden incluir otras variables como efectos por empresa en un determinado sector $y / o$ efectos temporales cuando existen datos panel; es decir, datos de corte transversal y de series de tiempo para una cohorte de datos.

\section{Modelo Econométrico}

Dado que se tienen datos desde 1995 hasta 2008 para 15 bancos, se utilizarán y analizarán los efectos y la eficiencia con modelos de datos panel; es decir, un modelo que utiliza simultáneamente corte transversal y datos de tiempo. Para datos panel hay dos metodologías: datos panel con efectos fijos $y$ datos panel con efectos aleatorios ${ }^{11}$. Los efectos fijos se utilizan cuando la muestra tomada es toda la población en una cohorte de datos. Los efectos aleatorios se utilizan cuando la muestra tomada es una proporción de la población total y por consiguiente se hacen inferencias hacia la población total a partir de la muestra.

En este trabajo la muestra corresponde a toda la población de bancos comerciales en Colombia, por lo tanto se utilizarán efectos fijos. Se utilizarán tres modelos con efectos fijos: el primero para medir los rendimientos a escala, el segundo con efectos por banco y el tercero con efectos temporales. En la literatura es más común utilizar simultáneamente efectos por empresa y efectos temporales; pero generalmente los resultados no varían mucho ${ }^{12}$.

\subsection{Rendimientos constantes}

Se utiliza el modelo (3) para medir los rendimientos a escala. La prueba que se hace es una prueba $\mathrm{F}^{13}$, en la cual la hipótesis nula afirma que la suma de las elasticidades es igual a uno y la hipótesis alterna que la suma de las elasticidades es menor que uno. En el primer caso habrá rendimientos constantes de escala y en el segundo rendimientos decrecientes de escala. El modelo econométrico que se utiliza para la prueba de rendimientos es el siguiente:

$$
\mathrm{LnY}_{\mathrm{it}}=\rho_{0}+\rho_{1} \operatorname{LnK}_{\mathrm{it}}+\rho_{2} \operatorname{LnL}_{\mathrm{it}}+v_{\mathrm{it}}
$$

Donde, el subíndice i corresponde al banco y el subíndice t al año, $L n Y_{i t}$ es el logaritmo natural de los ingresos e intereses de los bancos, $L_{n K} K_{i t}$ es el logaritmo natural de los activos de los bancos, en este trabajo los préstamos son considerados como los activos, $L n L_{i t}$ es el logaritmo natural de los sueldos y honorarios de los trabajadores, $\rho_{0}$ es la constante del modelo, $\rho_{1}$ es la elasticidad de los ingresos de los bancos con respecto a los activos de los mismos, $\rho_{2}$ es la elasticidad de los ingresos de los bancos con respecto a los sueldos y honorarios de los trabajadores, $y v_{i t}$ es el error de predicción. Los resultados de la regresión (3) se presentan en las Tablas. 1 y 2.

Tabla 1. Resultados de la regresión ${ }^{14}$

\begin{tabular}{|l|c|c|c|}
\hline & Coeficientes & Error típico & Estadístico t \\
\hline Intercepción & 0,78907354 & 0,17432861 & 4,5263572 \\
\hline LN K & 0,72624257 & 0,02576622 & 28,1858443 \\
\hline LN W & 0,25545462 & 0,02783858 & 9,17628113 \\
\hline
\end{tabular}

10 Se utiliza una prueba F, para determinar si existen rendimientos constantes de escala. Con esta prueba se mide la eficiencia de los factores utilizados sobre los ingresos.

11 En las regresiones se utiliza efectos fijos, dado que efectos aleatorios se utiliza cuando se tiene una submuestra de toda la población, para hacer inferencias para la población. En este caso la muestra es la población.

12 Modelo de datos panel con efectos temporales.Ver en "Análisis Econométrico" de William H. Green.

13 Ejemplos de la prueba F para rendimientos constantes se pueden ver en "Análisis Econométrico" de William H. Green.

14 Resultados de la investigación. 
Tabla 2. Resultados de la regresión ${ }^{15}$

\begin{tabular}{|l|c|c|c|}
\hline \multicolumn{4}{|c|}{ Matriz de Covarianzas } \\
\hline & constante & LnK & LnL \\
\hline constante & 0,030390463 & $-0,00133107$ & $-0,001247289$ \\
\hline LnK & $-0,00133107$ & 0,000663898 & $-0,00060667$ \\
\hline LnL & $-0,001247289$ & $-0,00060667$ & 0,000774986 \\
\hline
\end{tabular}

Los resultados de la prueba $\mathrm{F}$, de la Tabla 3 , que se infieren de las Tablas 1 y 2 , nos dice si realmente la suma de los coeficientes de los logaritmos del capital (préstamos) y trabajo (salarios) es uno o menor que uno.

Tabla 3. Resultados de la Prueba $F^{16}$

\begin{tabular}{|l|l|}
\hline \multicolumn{2}{|c|}{ Prueba F de rendimientos } \\
\hline F calculada & 1,485269799 \\
\hline F Tabla (95\%) & 3,84 \\
\hline F Tabla (99\%) & 6,63 \\
\hline
\end{tabular}

El valor del $\mathrm{F}$ calculado ${ }^{17}$ está en la hipótesis nula, tanto al 95 como al 99 por ciento de niveles de confianza, lo que significa que la suma de las elasticidades de los logaritmos del capital y el trabajo suma uno. Esto significa que en el sector bancario entre 1995 y 2008 existen rendimientos constantes de escala. Lo que a su vez significa que por cada uno por ciento que aumentan el capital (préstamos) y el trabajo (salarios), entonces los ingresos del sector bancario aumentan en un uno por ciento.

\subsection{Efectos por año}

La especificación del modelo de efectos fijos y efectos por año tiene la siguiente forma ${ }^{18}$ :

$$
\mathrm{LnY}_{\mathrm{it}}=\theta_{0}+\theta_{1} \mathrm{LnK}_{\mathrm{it}}+\theta_{2} \mathrm{LnL}_{\mathrm{it}}+\sum \delta_{\mathrm{t}} \mathrm{t}+\mu_{\mathrm{it}}
$$

Donde, el subíndice i corresponde al banco y el subíndice $\mathrm{t}$ al año, $\operatorname{Ln} Y_{i t}$ es el logaritmo natural de los ingresos e intereses de los bancos, $L n K_{i t}$ es el logaritmo natural de los activos de los bancos, en este trabajo los préstamos son considerados como los activos, $L n L_{i t}$ es el logaritmo natural de los sueldos $y$ honorarios de los trabajadores, $t$ es una dummie pare el t-ésimo año, $\theta_{0}$ es la constante del modelo, que según el paquete estadístico utilizado corresponde al año cuya dummie se omite en el modelo, $\theta_{1}$ es la elasticidad de los ingresos de los bancos con respecto a los activos de los mismos, $\theta_{2}$ es la elasticidad de los ingresos de los bancos con respecto a los sueldos y honorarios de los trabajadores, ${ }_{t}$ es el coeficiente correspondiente al t-ésimo año, $\mu_{i t}$ es el error correspondiente al i-ésimo banco y al t-ésimo año.

\subsection{Efectos por Empresa}

La especificación del modelo de efectos fijos y efectos por empresa tiene la siguiente forma ${ }^{19}$ :

$$
\operatorname{LnY}_{i t}=\beta_{0}+\beta_{1} \operatorname{LnK}_{i t}+\beta_{2} \operatorname{LnL}_{\mathrm{it}}+\sum \rho_{\mathrm{i}} \mathrm{B}_{\mathrm{i}}+\varepsilon_{\mathrm{it}}
$$

Donde, el subíndice i corresponde al banco y el subíndice $\mathrm{t}$ al año, $\operatorname{Ln} Y_{\text {it }}$ es el logaritmo natural de los ingresos e intereses de los bancos, $L_{n K}$ es el logaritmo natural de los activos fijos de los bancos, $\mathrm{LnL}_{i t}$ es el logaritmo natural de los sueldos y honorarios de los trabajadores, $B_{i}$ es una dummie pare el i-ésimo banco, $\beta_{0}$ es la constante del modelo, que según el paquete estadístico utilizado corresponde al banco cuya dummie se omite en el modelo, $\beta_{1}$ es la elasticidad de los ingresos de los bancos con respecto a los activos de los mismos, $\beta_{2}$ es la elasticidad de los ingresos de los bancos con respecto a los sueldos y honorarios de los trabajadores, $\rho_{i}$ es el coeficiente correspondiente al i-ésimo banco, y $\varepsilon_{i t}$ es el error correspondiente al i-ésimo banco y al t-ésimo año.

\footnotetext{
Resultados de la investigación.

Resultados de la investigación.

$\mathrm{F}=(0,7262+0,2554-1) /(0,00066+0,00077-2(0,0006066))$

Modelo de datos panel con efectos temporales. Ver "Análisis econométrico" de William H. Green.

9 Modelo de datos panel con efectos por empresa. Ver "Análisis econométrico" de William H. Green.
} 


\section{Resultados}

\subsection{Efectos por año}

Las estadísticas de regresión, el análisis de varianza y el resultado del modelo (4), de efectos por año se resumen en las Tablas 4 y 5 . De la Tabla 4 se puede destacar que el $\mathrm{R}$ cuadrado y el $\mathrm{R}$ cuadrado ajustado son muy altos, 0,95 en ambos casos, lo que significa que el 95\% de la variable ingresos se explica en un 95\% por las variables activos fijos (préstamos) y sueldos y honorarios. En la Tabla 5 se ratifica que el modelo es altamente significativo, al analizar el valor crítico de F se comprueba que el valor de la probabilidad es muy cercano a cero, lo que permite inferir que en conjunto el modelo de efectos por año es significativo con un nivel de confianza superior al 99,99\%.

Tabla 4. Coeficientes

\begin{tabular}{|l|l|}
\hline \multicolumn{2}{|c|}{ Estadísticas de la regresión } \\
\hline Coeficiente de correlación múltiple & 0,979228773 \\
\hline Coeficiente de determinación $\mathrm{R}^{\wedge} 2$ & 0,95888899 \\
\hline $\mathrm{R}^{\wedge} 2$ ajustado & 0,955591315 \\
\hline Error típico & 0,199216912 \\
\hline Observaciones & 203 \\
\hline
\end{tabular}

Tabla 5. Análisis de varianza

\begin{tabular}{|l|c|c|c|}
\hline & $\begin{array}{c}\text { Grados } \\
\text { de libertad }\end{array}$ & $\begin{array}{c}\text { Suma de } \\
\text { cuadrados }\end{array}$ & $\begin{array}{c}\text { Promedio de } \\
\text { los cuadrados }\end{array}$ \\
\hline Regresión & 15 & 173,1028423 & 11,54018949 \\
\hline Residuos & 187 & 7,42153971 & 0,039687378 \\
\hline Total & 202 & 180,524382 & \\
\hline
\end{tabular}

En la Tabla 6 se presentan los valores de los coeficientes del modelo (4). Hay que decir que no aparece el valor de la constante debido a que en este tipo de modelos de panel con efectos por año, al tener $\mathrm{n}$ años hay que introducir $\mathrm{n}-1$ dummies, $\mathrm{y}$ el valor de la constante es el coeficiente del año que se omitió. Este coeficiente se le suma a cada uno de los coeficientes de los otros años para obtener los coeficientes verdaderos por año, que son los que aparecen en la Tabla 6. El año que se omitió fue 2008, por lo tanto el coeficiente de este año es el valor de la constante. A partir de los datos encontrados se pueden hacer los siguientes análisis: El año en que los bancos fueron más eficientes fue 1995, el año en que los bancos fueron menos eficientes fue 2002, la recesión económica de 1999, durante la cual el crecimiento del PIB fue de $-4,2$ por ciento, afectó significativamente la eficiencia de los bancos entre 1999 y 2006.

Tabla 6. Coeficientes del Modelo20

\begin{tabular}{|c|c|}
\hline \multicolumn{2}{|c|}{ Variable dependiente: $\operatorname{Ln} Y$} \\
\hline $\operatorname{Ln} \mathrm{K}$ & 0,67522982 \\
\hline Ln L & 0,319951728 \\
\hline 1995 & 0,846451181 \\
\hline 1996 & 0,789497891 \\
\hline 1997 & 0,738151247 \\
\hline 1998 & 0,783821043 \\
\hline 1999 & 0,754455703 \\
\hline 2000 & 0,60726352 \\
\hline 2001 & 0,640999525 \\
\hline 2002 & 0,576741117 \\
\hline 2003 & 0,593511007 \\
\hline 2004 & 0,625388544 \\
\hline 2005 & 0,643431723 \\
\hline 2006 & 0,652624163 \\
\hline 2007 & 0,721226932 \\
\hline 2008 & 0,725098318 \\
\hline
\end{tabular}

20 Resultados de la investigación. 
Tabla 7. Prueba $F^{21}$

\begin{tabular}{|l|r|}
\hline \multicolumn{2}{|c|}{ Diferencia entre años } \\
\hline F calculada & 2,245219976 \\
\hline F Tabla(.95) & 1,75 \\
\hline F Tabla(.99) & 2,18 \\
\hline
\end{tabular}

Por otro lado, según la Tabla 7, la prueba $\mathrm{F}^{22}$ comprueba que hay diferencia entre las eficiencias de los años, tanto al 99\% como al 95\%. En ambos casos se rechaza la hipótesis nula de igualdad entre años y se acepta la alterna de diferencia entre años. Pero, el valor $\mathrm{F}$ calculado está muy cercano a los valores límite de la Tabla, entonces no se puede decir que exista una diferencia significativa entre la eficiencia por años.

\subsection{Efectos por banco}

Las estadísticas de regresión, el análisis de varianza y el resultado del modelo (5) de efectos por banco se resumen en las Tablas 8, 9, 10 y 11 . El coeficiente de determinación es cercano a uno, lo que indica que el 98,3\% de la variable ingresos e intereses está determinada por las variables préstamos y salarios en el conjunto de todos los bancos. En la Tabla 9 se comprueba que el modelo es altamente significativo, al analizar el valor crítico de $\mathrm{F}$ se observa que el valor de la probabilidad es muy cercano a cero, lo que indica que en conjunto el modelo de efectos por año es significativo con un nivel de confianza superior al 99,99\%.

Tabla 8. Datos de la regresión ${ }^{23}$

\begin{tabular}{|l|r|}
\hline Coeficiente de correlación múltiple & 0,991886234 \\
\hline Coeficiente de determinación $\mathrm{R}^{\wedge} 2$ & 0,983838301 \\
\hline $\mathrm{R}^{\wedge} 2$ ajustado & 0,982448047 \\
\hline Error típico & 0,125243434 \\
\hline Observaciones & 203 \\
\hline
\end{tabular}

1 Resultados de la investigación.

Ver "Análisis econométrico" de William H. Green.

Resultados de la investigación.

24 Resultados de la investigación.

25 Se omite la constante, dado que para evitar colinealidad perfecta se omite la dummy del banco Sudameris, el valor de la constante corresponde entonces al Banco Sudameris.
Tabla 9. Análisis de varianza ${ }^{24}$

\begin{tabular}{|l|c|c|c|c|c|}
\hline & $\begin{array}{c}\text { Grados de } \\
\text { libertad }\end{array}$ & $\begin{array}{c}\text { Suma de } \\
\text { cuadrados }\end{array}$ & $\begin{array}{c}\text { Promedio de } \\
\text { los cuadrados }\end{array}$ & $\mathbf{F}$ & $\begin{array}{c}\text { Valor } \\
\text { crítico } \\
\text { de F }\end{array}$ \\
\hline $\begin{array}{l}\text { Regre- } \\
\text { sión }\end{array}$ & 16 & 177,6068013 & 11,10042508 & 707,668 & $3,5044 \mathrm{E}-157$ \\
\hline $\begin{array}{l}\text { Resi- } \\
\text { duos }\end{array}$ & 186 & 2,917580718 & 0,015685918 & & \\
\hline Total & 202 & 180,524382 & & & \\
\hline
\end{tabular}

La Tabla 10 corresponde al modelo $(5)^{25}$, se puede observar que los bancos más eficientes son el banco Agrario de Colombia y el banco de Occidente, y los menos eficientes son Amrobank y el Banco Caja Social-Colmena BCSC. Una posible explicación de por qué el Banco Agrario es el más eficiente en cuanto a retornos sobre los ingresos de los préstamos y los salarios, es que su única función son los préstamos y no tiene otro tipo de inversión que le genere rentabilidad, en ese sentido es el más eficiente, porque su función es esa específicamente. Por otro lado, el Banco Caja Social Colmena es el menos eficaz, en cuanto al uso de los factores y su efecto en los ingresos. La posible explicación es que por la misma función social de la Caja, las tasas de interés que cobran por los créditos son menores que el resto del sistema financiero y por lo tanto menores los retornos de los factores frente a los ingresos.

La Tabla 11 presenta el valor calculado del estadístico F para la prueba de hipótesis de diferencias entre bancos. El valor $\mathrm{F}$ calculado es muy superior a los valores críticos de la F, tanto al 99\% como al 95\%. Esto lo que significa es que se rechaza la hipótesis nula de igualdad entre bancos y se acepta la alterna de diferencia significativa entre las eficiencia de los bancos. Como la diferencia entre los valores $F$ calculado y críticos es bastante grande, entonces se puede afirmar que la diferencia entre las eficiencias de los bancos de la muestra es bastante significativa, cosa que no se puede afirmar con tanta fuerza para la diferencia entre años. 
Tabla 10. Eficiencia de los bancos ${ }^{26}$

\begin{tabular}{|l|c|}
\hline \multicolumn{2}{|c|}{ Variable dependiente: Ln Y } \\
\hline Ln K & 0,817749005 \\
\hline Ln L & 0,199745486 \\
\hline Amrobank & 0,167832678 \\
\hline Bancolombia & 0,207736742 \\
\hline Bogotá & 0,231210658 \\
\hline Crédito & 0,309323435 \\
\hline Popular & 0,22946683 \\
\hline Banagrario & 0,937400747 \\
\hline BBVA & 0,255135183 \\
\hline Citybank & 0,329913675 \\
\hline HSBC & 0,29789304 \\
\hline Santander & 0,200635057 \\
\hline AV Villas & 0,19477127 \\
\hline BCSC & 0,113693156 \\
\hline Colpatria & 0,331230635 \\
\hline Occidente & 0,540818275 \\
\hline Sudameris & 0,309994701 \\
\hline
\end{tabular}

Tabla 11. Prueba F para diferencia entre bancos ${ }^{27}$

\begin{tabular}{|l|r|}
\hline \multicolumn{2}{|c|}{ Diferencia entre bancos } \\
\hline$F$ (calculada) & 25,89491249 \\
\hline$F($ Tabla-99\%) & $2,0^{\prime} 7$ \\
\hline$F($ Tabla-95\%) & 1,77 \\
\hline
\end{tabular}

\section{Conclusiones}

De los resultados del trabajo, se puede decir que el sector financiero presenta rendimientos constantes a escala tanto en el modelo de efectos por año como en el de efectos por banco; esto lo que significa es que en el periodo analizado (1995-2008), por cada uno por ciento que aumentan los factores de producción, en este caso los préstamos y los salarios, entonces los ingresos de los bancos aumentan en un uno por ciento, para el conjunto de quince bancos de la muestra.

La elasticidad de los ingresos frente a los préstamos en el modelo de efectos por año es 0,67, lo que indica que por cada uno por ciento que aumentan los préstamos los ingresos de los bancos aumentan en un 0,67 por ciento. En el modelo de efectos por banco esta misma elasticidad es de 0,81. La elasticidad de los salarios frente a los ingresos en el modelo de efectos por año es 0,32 , lo que significa que por cada uno por ciento que aumentan los salarios entonces el ingreso aumenta un 0,32 por ciento. En el modelo de efectos por banco esta misma elasticidad es 0,2. En los dos modelos el aporte de los préstamos es mucho más significativo que el aporte de los salarios de los trabajadores. El aporte de los préstamos es mayor que el aporte de los salarios sobre los ingresos debido a que, en últimas, los ingresos dependen de los préstamos.

La recesión económica golpeó fuertemente la eficiencia bancaria entre los años 1999 y 2006. Esto se evidencia en el modelo de efectos por año. El banco más eficiente en cuanto a los retornos de los préstamos y salarios sobre los ingresos es el banco Agrario y le sigue el banco de Occidente. Los menos eficientes son Amrobank y el Banco CajaSocial-Colmena. La explicación del por qué el Banco Agrario es el que reporta mayores retornos, especialmente de los préstamos hacia sus ingresos, es que éste banco fue creado precisamente después de la crisis financiera de 1999 y su fin último se enfoca solamente en los préstamos al sector agrario, entonces los retornos de éstos préstamos son más eficientes que otros bancos que se enfocan en otro tipo de inversiones de portafolio.

Hay una diferencia bastante significativa en la eficiencia, medida por los retornos, al compararse entre bancos. También hay una diferencia al compararse por años, pero no es muy significativa. Lo

\footnotetext{
26 Resultados de la investigación.

27 Resultados de la investigación.
} 
que significa que no son comparables los bancos en cuanto al uso eficiente de los factores, ni tampoco los años son comparables; aunque hay mayor similitud en las eficiencias al compararlas por años que al compararlas por bancos.

\section{Referencias}

Bailey, M, Ghost, D \& Tanna, S. (2002). Thai Financial Sector Efficiency Prior to the East Asian Financial Crisis. School of Economics \& Politics. University of Ulster. Jordanstown. UK

Bernal, O \& Herrera, S (1983). "Producción, Costos y Economías de escala en el Sistema Bancario Colombiano". Ensayos sobre política económica. Banco de la República. En: Revista ESP, 3: 7-36.

Berrio, D \& Muñoz, A. (2005). "Análisis de la Eficiencia Relativa del Sistema Bancario en Colombia en el Período 1993-2003 y Propuesta Estratégica de Fortalecimiento". En: Revista Pensamiento y Gestión, 18: 1-30.

Carvajal, A \& Zuleta, H. (1997). "Desarrollo del Sistema Financiero y Crecimiento Económico". Borradores de Economía. No. 67: 1-37.

Castro, A. (2001). "Eficiencia-X en el Sector Bancario Colombiano". Documento 158. Archivos de economía de la Dirección de Estudios Económicos. Departamento Nacional de Planeación.

Chai, H \& Rhee, Y. (2005). "Financial Integration and Financial Efficiency in East Asia". Kyonggi University. Paper presented at the Claremont-KIEP international Conference in November 2005.
Ferrufino, A. (1991). "Reestimación y Ampliación de la Evidencia Sobre las Economías de Escala en el Sistema Financiero Colombiano". Ensayos sobre política económica. Banco de la República. En: Revista ESP, 19: 69-94.

Friffill, J. (2003)."Growth and Finance". School of Economics, Mathematics, and Statistics, Birkbeck College, University of London. Volumen 26, número 2: 113-136.

Gómez, J. (2001). "Proceso de Consolidación del Sistema Bancario: Fusiones, Rentabilidad y Competencia 1994-2000". Concurso de Investigación para Jóvenes economistas. Banco Central de Reserva del Perú.

Green, W. (2001) Análisis Econométrico. Editorial Prentice Hall. Capítulo 14.

Janna, M. (2003). "Eficiencia en Costos, cambios en las condiciones generales del mercado y crisis en la banca Colombiana: 1992-2002"

Levine, R. (2005). "Finance and Growth: Theory and Evidence". Department of Economics. Brown University.

Sarmiento, J \& Ramírez, A. (2005)"Los Costos de la Desinflación en Colombia según el modelo de Buiter-Miller", Cuadernos de Economía, 43: 129-159

Suescun, R. (1987). "Nueva Evidencia Sobre Economías de escala en la Banca Colombiana". Ensayos sobre política económica. Banco de la República. En: Revista ESP, 12: 5-22.

Suescun, R \& Misas, M. (1996). "Cambio Tecnológico, Ineficiencia de Escala e Ineficiencia X en la Banca Colombiana". Borradores de Economía. No. 59: 1-31.

Terada, A. (2002). "Desarrollo Financiero y Ciclos Reales" Economía Internacional, Estudios Recientes. No 796: 163-170.

Wu, S. (2002). "Productivity and Efficiency Analysis of Australia Banking Sector under Deregulation". School of Accounting, Economics and Finance. Deakin University 